\title{
Insegurança alimentar entre adolescentes brasileiros: um estudo de validação da Escala Curta de Insegurança Alimentar
}

\author{
Household food insecurity in Brazilian \\ adolescents: A validation study
}

\author{
Stefanie Eugênia dos Anjos Campos COELHO ${ }^{1}$ \\ Rodrigo Pinheiro de Toledo VIANNA ${ }^{2}$ \\ Ana Maria SEGALL-CORREA ${ }^{3}$ \\ Rafael PEREZ-ESCAMILLA ${ }^{4}$ \\ Muriel Bauermann GUBERT ${ }^{5}$
}

\section{R E S U M O}

\section{Objetivo}

Os objetivos do trabalho foram avaliar a validade interna e a capacidade preditiva da escala de segurança alimentar de seis itens aplicada a adolescentes.

\section{Métodos}

Foi um estudo transversal com amostra representativa de adolescentes brasileiros ( $N=14690$ ), realizado em escolas públicas e privadas nas 26 capitais de estados brasileiros e no Distrito Federal por meio de questionário online.

\section{Resultados}

A maior parte dos respondentes era do sexo feminino (53,2\%), com idade média de 14,4 anos, sendo $72,7 \%$ de escolas públicas. O comportamento da escala, observado pelo modelo de Rasch, foi melhor sem o item cinco, apresentando valores ótimos de Infit e nível de severidade crescente entre os itens. O alfa de Cronbach foi 0,77 , e as análises do funcionamento diferencial dos itens mostraram comportamento dos itens semelhante entre os subgrupos avaliados. A análise fatorial mostrou a unidimensionalidade do instrumento.

\footnotetext{
1 Universidade de Brasília, Departamento de Nutrição, Programa de Pós-Graduação em Nutrição Humana. Brasília, DF, Brasil.

2 Universidade Federal da Paraíba, Centro de Ciências da Saúde, Departamento de Nutrição. João Pessoa, PB, Brasil.

${ }^{3}$ Universidade de Campinas, Faculdade de Ciência Médicas, Pós-Graduação em Saúde Coletiva. Campinas, SP, Brasil.

${ }^{4}$ Yale School of Public Health, Course of Epidemiology. New Haven, CT, United States of America.

5 Universidade de Brasília, Faculdade de Ciências da Saúde, Departamento de Nutrição. Campus Darcy Ribeiro, Asa Norte, 70910-900, Brasília, DF, Brasil. Correspondência para/Correspondence to: MB GUBERT. E-mail: <murielgubert@gmail.com>. Apoio: Conselho Nacional de Desenvolvimento Científico e Tecnológico (Processo nº 559384/2010-6), Ministério da Educação e Secretarias de Educação.
} 


\section{Conclusão}

Foi proposta a retirada do item cinco e novos pontos de corte para a escala curta de segurança alimentar. A escala curta de segurança alimentar é válida e confiável para mensurar insegurança alimentar domiciliar entre adolescentes brasileiros.

Palavras-chave: Adolescentes. Modelo de Rasch. Segurança Alimentar.

\section{A B S T R A C T}

\section{Objective}

The objective of this study was to evaluate the internal and predictive validity of a six-item household food insecurity scale to measure food insecurity in Brazilian adolescents.

\section{Methods}

A cross-sectional study with a representative sample of Brazilian adolescents $(N=14,690)$ was conducted in public and private schools in 26 state capitals and in the Federal District of Brazil using an online questionnaire.

\section{Results}

Most respondents were female (53.2\%) with mean age of 14.4 years, and $72.7 \%$ of them attended public schools. The psychometric properties of the scale, assessed using the Rasch model, showed better response rates without the item five, exhibiting optimal Infit statistics and an increasing level of severity among the items. Cronbach's á was 0.767. Differential item functioning analyses showed similar behavior between the items in the subgroups evaluated. Factor analysis confirmed the unidimensionality of the scale.

\section{Conclusion}

This study proposes the removal of the item five and the inclusion of new cut-off points for this short form of the scale. The Household Food Security Scale (short form) is valid and reliable to measure household food insecurity in Brazilian adolescents.

Keywords: Adolescents. Rasch model. Food Security.

\section{N T R O D U Ç Ã O}

A insegurança alimentar configura-se como a violação do direito humano à alimentação adequada, manifestando-se de diversas formas, desde uma situação de desconforto psicológico, como ansiedade devido à preocupação que o alimento venha a faltar no domicílio por falta de recursos para sua aquisição, até uma dimensão concreta e física, caracterizada por restrições quantitativas de alimentos, que compromete e coloca em risco a saúde e o bem-estar das pessoas ${ }^{1}$.

A segurança alimentar e nutricional pode ser avaliada por indicadores que mensuram indiretamente a vulnerabilidade da família à situação de fome. Esses indicadores estão associados à ocorrência de insegurança alimentar nos domicílios, como: disponibilidade de alimentos (na- cional, regional ou local), consumo calórico médio, rendimento familiar, consumo alimentar e dados antropométricos. Esses indicadores indiretos, entretanto, referem-se, em geral, aos determinantes de insegurança alimentar e nutricional ou as suas consequências para a saúde dos indivíduos, sendo, portanto, limitados para a apreensão do fenômeno da insuficiência ou falta de acesso aos alimentos ${ }^{2,3}$.

Com intuito de mensurar diretamente a insegurança alimentar, foi desenvolvido pelo United States Department of Agriculture (USDA, Departamento de Agricultura dos Estados Unidos) um questionário com 18 itens capaz de medir diretamente a insegurança alimentar e a fome ${ }^{4}$. No Brasil, a escala americana foi adaptada e validada, dando origem à Escala Brasileira de Medida de Insegurança Alimentar (EBIA), que classifica o domicílio em seguro ou inseguro em três níveis: leve, moderado e grave ${ }^{5,6}$. 
Ainda nos Estados Unidos, a partir da escala completa de 18 itens, foi desenvolvida uma versão abreviada do instrumento, com seis itens ${ }^{7}$, todos referentes à situação dos adultos residentes nos domicílios. Essa escala curta já foi utilizada no Brasil para a medida domiciliar de segurança alimentar ${ }^{8}$. Há referências, em outros países, de sua aplicação em população de adolescentes como instrumento alternativo de rastreamento de insegurança alimentar ${ }^{9,10}$. No entanto, seu uso com os adolescentes não foi validado em nenhum país da América Latina.

A situação de insegurança alimentar vivenciada na adolescência é relevante uma vez que essa fase é marcada por intenso desenvolvimento físico, cognitivo e emocional ${ }^{11-13}$. É de extrema importância o acesso contínuo à alimentação em quantidade suficiente e qualidade adequada para garantir o bom crescimento e desenvolvimento, não comprometendo o resultado final desse processo $^{14}$.

O presente trabalho tem como objetivo, portanto, avaliar a validade interna da escala de segurança alimentar de seis itens quando aplicada ao público adolescente, bem como sua capacidade preditiva da segurança ou insegurança alimentar nos domicílios.

\section{MÉ TOD OS}

Este estudo é parte da pesquisa "Mapeamento da cultura alimentar da população adolescente nas capitais brasileiras e Distrito Federal", financiada pelo Conselho Nacional de Desenvolvimento Científico e Tecnológico (Processo $n^{\circ}$ 559384/2010-6). Trata-se de um estudo transversal realizado com estudantes do $9^{\circ}$ ano do ensino fundamental em escolas públicas e privadas nas 26 capitais brasileiras e no Distrito Federal nos anos de 2011 e 2012.

O tamanho final da amostra foi de 15084 adolescentes, sendo realizada amostragem por conglomerados, com sorteio das escolas em um único estágio, de forma a gerar dados represen- tativos para a população adolescente residente nas capitais dos estados brasileiros e Distrito Federal. Para o cálculo do tamanho da amostra, utilizou-se nível de 95\% de confiança e erro aceitável de cinco pontos percentuais.

O número de estudantes pesquisados foi calculado com base no número total de adolescentes do Brasil e de sua distribuição nas cidades estudadas ${ }^{15}$. As escolas públicas e particulares que informaram possuir turmas de $9^{\circ}$ ano do ensino fundamental e laboratório de informática no censo escolar de 2010 foram ordenadas em uma lista, para sorteio aleatório. As escolas eram convidadas via e-mail e telefone para a participação na pesquisa, uma a uma, até que se alcançasse o número de alunos estimado para cada capital.

Foi desenvolvido um software interativo, composto por perguntas fechadas e autoadministradas pelos adolescentes, em plataforma online. A coleta de dados foi realizada entre setembro de 2011 e novembro de 2012. Todas as capitais foram visitadas previamente pela equipe da pesquisa, que instruía os representantes das escolas selecionadas quanto à aplicação do questionário online.

A pesquisa foi aprovada pelo Comitê de Ética em Pesquisa da Universidade de Brasília em junho de 2011 sob o registro de $n^{\circ}$ 034/11. Os pais ou responsáveis pelos alunos participantes assinaram o Termo de Consentimento Livre e Esclarecido, e as instituições de ensino concordaram com o uso do ambiente escolar para realização da pesquisa por meio de Termo de Ciência Institucional.

No presente estudo, foram utilizados dados relacionados à situação de segurança alimentar e nutricional nos domicílios onde residiam os adolescentes. Esses dados foram coletados utilizando-se a escala de segurança alimentar com seis itens, derivada do Household Food Security Survey Module (HFSSM), já traduzida e utilizada no Brasil, entretanto sem validação ${ }^{8}$. Essa versão traduzida por Santos et al. ${ }^{8}$ contém seis questões referentes à alimentação no domicílio nos últimos 12 meses e teve sua redação adaptada para 
melhor compreensão dos adolescentes (Quadro 1). As questões apresentam nível de severidade crescente, abrangendo situações desde não se ter uma alimentação variada (item 1) até a ocorrência de fome por falta de dinheiro (item 6) ${ }^{7}$.

A escala originalmente gera um escore de 0 a 6 , calculado pela soma das respostas afirmativas. Para as quatro primeiras e sexta questões, a resposta afirmativa corresponde a um ponto. Já para o quinto item da escala, que se refere à frequência do evento citado na questão quatro, a pontuação obedece ao seguinte critério: quando ocorre "muitas vezes" ou "algumas vezes", soma-se um ponto; quando ocorre "poucas vezes" ou "não sei", não há pontuação. Após a soma dos pontos e a formação do escore, os domicílios que atingem até um ponto são considerados em situação de segurança alimentar. São classificados como insegurança alimentar sem fome aqueles que somam entre dois e quatro pontos; e insegurança alimentar com fome quando a pontuação atinge cinco ou seis pontos ${ }^{7}$.

O banco de dados era gerado automaticamente após cada aluno inserir suas respostas, por alimentação eletrônica imediata, no servidor de hospedagem da pesquisa. Após a coleta, os dados foram migrados para o programa Statistical Package for the Social Sciences (SPSS Inc., Chicago, Estados Unidos) para Windows ${ }^{\circledR}$ versão 17, no qual foram realizados as análises descritivas, o cálculo do Qui-quadrado, alfa de Cronbach e análise fatorial da escala.

Foi verificada a associação entre insegurança alimentar domiciliar e as variáveis sexo, tipo de escola e escolaridade materna, mediante aplicação do teste Qui-quadrado, para averiguar o comportamento da escala.

Para verificar a consistência interna entre as respostas aos itens da escala e indicar se as respostas estavam correlacionadas entre si, foi calculado o coeficiente de alfa de Cronbach, que pode assumir valores entre 0 e 1: quanto mais próximo a 1, maior a confiabilidade das respostas aos itens da escala. De acordo Corrar et al. ${ }^{16}$, o valor mínimo aceitável de alfa é 0,7.

A análise fatorial é um dos procedimentos estatísticos mais utilizados na construção, revisão e avaliação de instrumentos psicométricos. Pode ser utilizada na verificação da unidimensionalidade, quando todos os itens de um instrumento estão medindo um único construto ou fator, e também no processo de validação de instrumentos. Neste estudo, foi feita análise fatorial para verificar se o instrumento media apenas um construto, ou seja, a insegurança alimentar. Tal fato é avaliado pelos autovalores de cada componente e pelo total da variância explicada por cada um, sendo que o número de fatores que o instrumento mensura é o número de componentes com autovalor maior que um ${ }^{17,18}$.

Quadro 1. Itens utilizados na versão curta da Escala de Segurança Alimentar adaptada de Bickel et al. ${ }^{7}$.

\begin{tabular}{|c|c|c|}
\hline Itens & Respostas & Pontos \\
\hline \multirow{2}{*}{$\begin{array}{l}\text { 1. Na sua casa, alguém deixou de ter uma alimentação variada, com frutas, saladas, feijão, arroz e } \\
\text { carne, porque não tinha dinheiro para comprar? }\end{array}$} & $\operatorname{sim}$ & 1 \\
\hline & Não & 0 \\
\hline \multirow[t]{2}{*}{ 2. Alguma vez a comida da sua casa terminou e não havia dinheiro para comprar mais? } & Sim & 1 \\
\hline & Não & 0 \\
\hline \multirow{2}{*}{$\begin{array}{l}\text { 3. Nos últimos } 12 \text { meses, você ou alguma outra pessoa na sua casa teve que comer menos ou deixou } \\
\text { de fazer alguma refeição por falta de dinheiro para comprar comida? }\end{array}$} & Sim & 1 \\
\hline & Não & 0 \\
\hline \multirow{2}{*}{$\begin{array}{l}\text { 4. Nos últimos } 12 \text { meses, você já comeu menos do que deveria, porque não havia dinheiro suficiente } \\
\text { para comprar comida? }\end{array}$} & Sim & 1 \\
\hline & Não & 0 \\
\hline \multirow[t]{2}{*}{$\begin{array}{l}\text { 5. Quantas vezes aconteceu de você comer menos do que deveria, porque não havia dinheiro suficien- } \\
\text { te para comprar comida, nos últimos } 12 \text { meses? }\end{array}$} & $\begin{array}{l}\text { Muitas vezes/ } \\
\text { Algumas vezes/ }\end{array}$ & 1 \\
\hline & Poucas vezes/Não sei & 0 \\
\hline \multirow{2}{*}{$\begin{array}{l}\text { 6. Nos últimos } 12 \text { meses, alguma vez você sentiu fome, mas não comeu, porque a sua família não } \\
\text { pôde comprar comida suficiente? }\end{array}$} & Sim & 1 \\
\hline & Não & 0 \\
\hline
\end{tabular}


O modelo de Rasch foi utilizado para avaliar a validade interna da versão curta da escala americana HFSSM. No caso da escala de segurança alimentar, o modelo espera que as famílias em segurança alimentar tenham menor probabilidade de responder aos itens positivamente, enquanto as famílias que experimentam insegurança são mais propensas a afirmá-los ${ }^{19}$. Esse modelo gera dois conjuntos de estatísticas a fim de avaliar a validade interna da escala: os valores de severidade e as estatísticas de ajuste ou estatísticas Fit ${ }^{20}$.

As estatísticas Fit são a razão do quadrado da diferença entre as estimativas esperadas e as observadas e são identificadas como médias do quadrado dos resíduos ${ }^{21}$. Caso a escala meça apenas um constructo, ela pode ser avaliada principalmente pelo valor Infit. Os valores esperados para a estatística Infit (valor médio quadrático) são: entre 0,7 e 1,3 considerados adequados e entre 0,8 e 1,2 considerados valores ótimos de ajuste da resposta estimada com a esperada ${ }^{20}$.

Este estudo comparou a severidade do item entre vários subgrupos e a amostra completa para verificar se havia diferenças baseadas nas características de cada subpopulação. Para isso foi realizada análise do Differential Item Functioning (DIF, Funcionamento Diferencial dos Itens). Os subgrupos analisados foram: feminino/ masculino; escola pública/privada; regiões Norte/ Sul e níveis de escolaridade materna analfabeta/ nível superior.

Análises pelo modelo de Rasch foram realizadas com o software Winsteps, versão 3.72. Foram feitas as seguintes análises: a) estimativa dos valores de severidades do item, b) estimativa dos valores de Infit de cada item, c) realização de análises DIF que comparam os subgrupos em relação ao total da amostra.

As análises foram realizadas em duas etapas: a primeira com a escala de seis itens de acordo com a classificação original, e a segunda considerando que o item 5 é um subitem da questão anterior e não um item independente. Foram exploradas duas opções de análise, retirando-se esse item e mesclando-o com o item 4.

\section{RES U L T A D OS}

Participaram da pesquisa 15084 adolescentes em todas as capitais brasileiras e Distrito Federal. Foram excluídos 394 indivíduos por falta de resposta em um ou mais itens da escala de segurança alimentar, totalizando 14690 sujeitos para a análise. Dos respondentes, 7822 eram do sexo feminino $(53,2 \%)$ e 6868 do sexo masculino (46,8\%), com média de idade de 14,4 anos (Desvio-Padrão-DP=1,15). A maioria dos alunos era de escolas públicas ( $n=10680 ; 72,7 \%$ ). Em relação à escolaridade materna, aproximadamente $41,0 \%$ tinham ou estavam cursando o ensino superior, e apenas 3,0\% eram analfabetas.

De acordo com a classificação original preconizada para a escala de seis itens, grande parte dos domicílios dos participantes estava com segurança alimentar $(88,1 \%)$. A insegurança alimentar foi prevalente em $11,9 \%$ dos domicílios, sendo que 2,6\% estavam em situação de insegurança alimentar com episódios de fome. Quando os dados de segurança alimentar foram analisados segundo as características da amostra, observou-se que a insegurança alimentar foi mais prevalente em domicílios com adolescentes do sexo masculino, provenientes de escolas públicas e filhos de mães com menor escolaridade. As diferenças encontradas foram estatisticamente significativas (Tabela 1).

A análise de consistência interna da escala com seis itens mostrou um alfa de Cronbach igual a 0,79 . Mediante análise fatorial, foi observado que $53,5 \%$ do total da variância podem ser explicadas por um único fator, o único com autovalor (eigenvalue) maior que um (2 675). Todas as cargas fatoriais do fator extraído foram acima de 0,32; dessa forma, pode-se afirmar que os itens da escala compartilham um único fator, ou seja, a escala mede apenas um construto, no caso, a insegurança alimentar. 
Os resultados da análise pelo modelo de Rasch mostraram que a escala com seis itens apresenta severidade crescente a cada item, porém o item número 5, que se refere à frequência do evento "comer menos por falta de dinheiro", perguntado no item anterior, aparece com maior severidade do que o último item, referente à ocorrência de fome no domicílio. Esse mesmo comportamento permaneceu quando os itens 4 e 5 foram mesclados em um único item. $\mathrm{Na}$ análise da escala sem o item cinco, a gravidade entre os itens foi crescente, desde a falta de uma alimentação variada até a ocorrência de fome, conforme esperado (Figura 1).

Os valores de Infit para cada item não ultrapassaram os valores mínimo e máximo aceitáveis $(0,7$ e 1,3$)$, mostrando que a escala tem um bom ajuste entre a resposta estimada e a resposta esperada pelo modelo. Quando retirada a questão 5, o ajuste passa a ser considerado ótimo, valores entre 0,8 e 1,2, com valor mínimo igual a 0,98 e máximo igual a 1,17 (Figura 2).

Uma vez que o comportamento da escala era melhor sem o item cinco, foi refeito o alfa de

Tabela 1. Situação de segurança alimentar segundo as características dos adolescentes nas capitais brasileiras e do funcionamento diferencial dos itens. 2013.

\begin{tabular}{|c|c|c|c|c|c|}
\hline Característica & $\mathrm{N}$ & SA & IA sem fome & IA com fome & Valor de $p^{*}$ \\
\hline \multicolumn{6}{|l|}{ Sexo } \\
\hline Feminino & 7822 & 89,2 & 8,4 & 2,3 & \multirow[t]{2}{*}{$<0,001$} \\
\hline Masculino & 6868 & 86,9 & 10,3 & 2,9 & \\
\hline \multicolumn{6}{|l|}{ Tipo de escola } \\
\hline Pública & 10684 & 86,0 & 11,1 & 3,0 & \multirow[t]{2}{*}{$<0,001$} \\
\hline Privada & 4006 & 93,9 & 4,6 & 1,5 & \\
\hline \multicolumn{6}{|l|}{ Escolaridade materna } \\
\hline Analfabeta & 388 & 70,6 & 15,5 & 13,9 & \multirow[t]{4}{*}{$<0,001$} \\
\hline Ensino Fundamental & 2814 & 84,1 & 12,2 & 3,7 & \\
\hline Ensino Médio & 3994 & 88,6 & 9,5 & 1,9 & \\
\hline Ensino Superior & 5123 & 92,2 & 6,3 & 1,6 & \\
\hline
\end{tabular}

Nota: ${ }^{*}$ Teste Qui-quadrado.

SA: Segurança Alimentar; IA: Insegurança Alimentar.
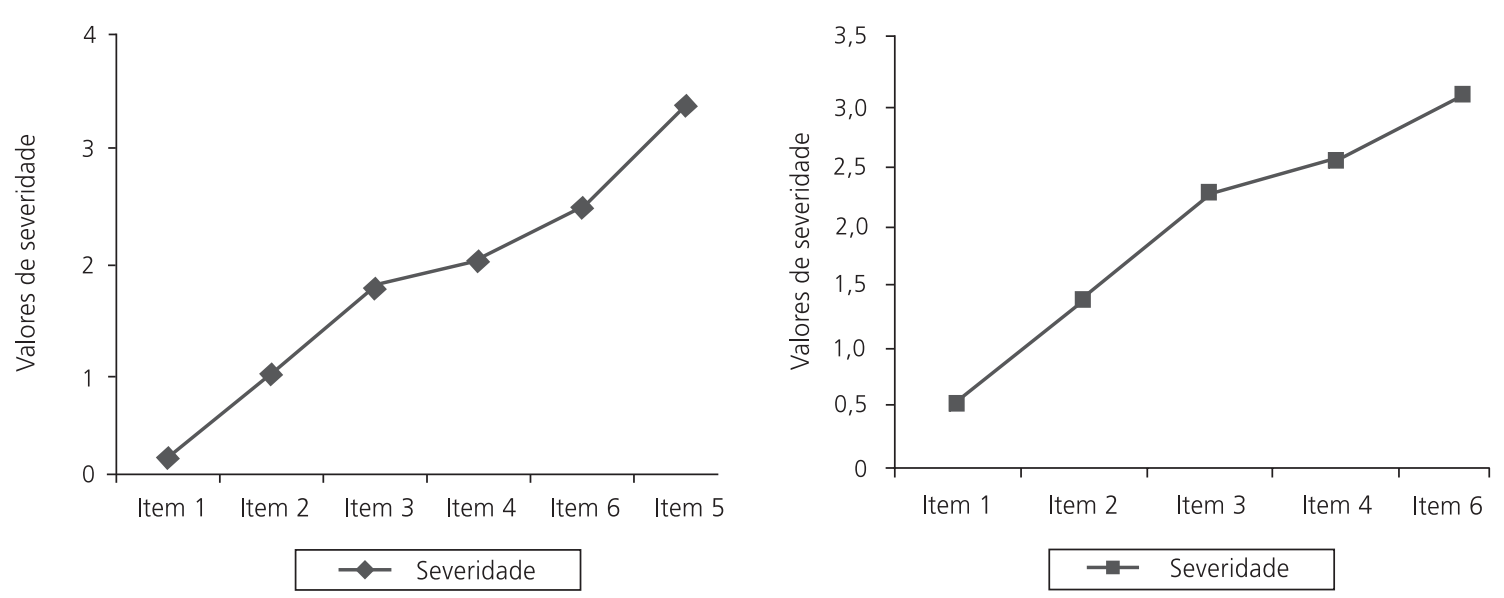

Figura 1. Comparação dos valores de severidade dos itens da escala de segurança alimentar reduzida com seis itens e a escala sem o item cinco em adolescentes brasileiros, Brasil ( $n=14$ 680).

Nota: Os valores de severidade são apresentados em unidades logarítmicas: cada unidade representa dez vezes mais a magnitude da severidade. 

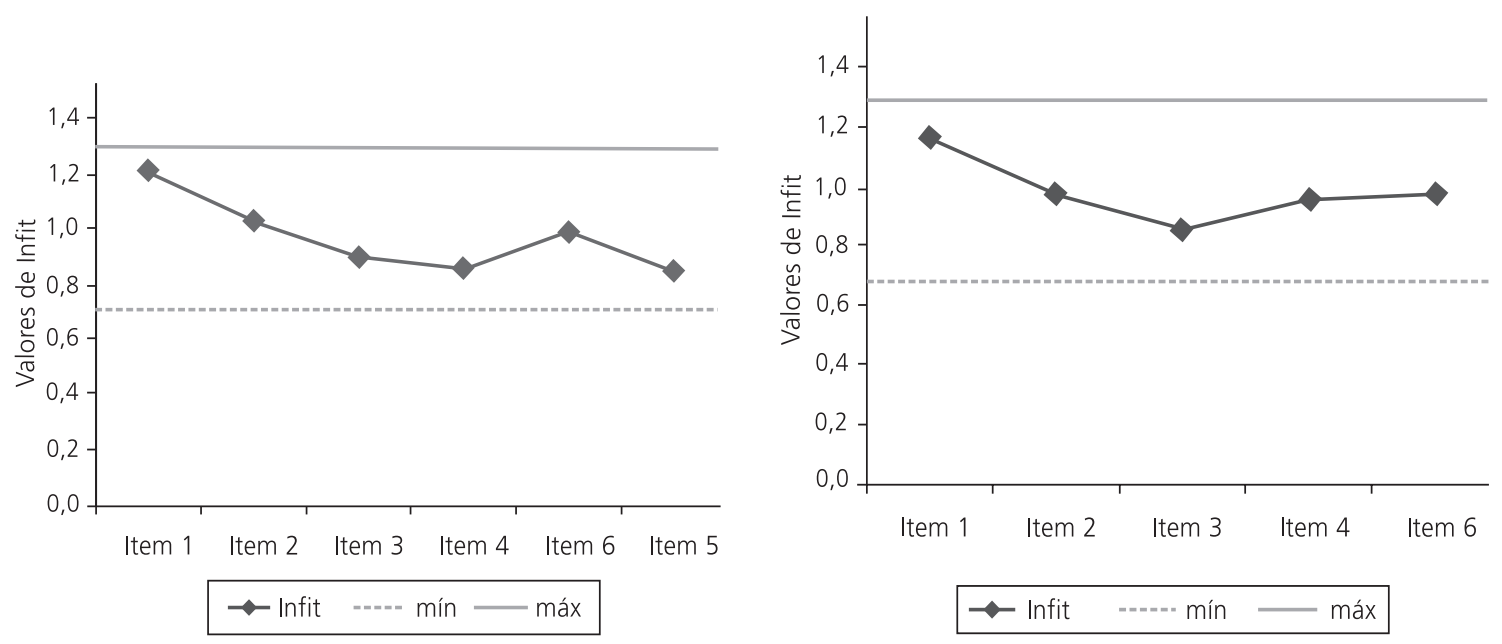

Figura 2. Comparação entre os valores de Infit dos itens da escala de segurança alimentar reduzida com seis e cinco itens em adolescentes brasileiros, Brasil ( $n=14$ 680).

Nota: Mín: Mínimo; Máx: Máximo.

Cronbach, que apresentou um novo valor de 0,77. Contando que a escala tem melhores severidade e ajuste quando retirado o item cinco, optou-se por realizar as análises seguintes sem esse item.

As análises do funcionamento diferencial dos itens mostraram que o funcionamento dos itens é semelhante entre os subgrupos avaliados. Os valores de DIF para todos eles foram inferiores a 0,5 . Tais resultados revelam que, independentemente do grupo testado, o comportamento da escala é sempre válido. A Figura 3 mostra os subgrupos com menor e maior distância entre a severidade dos itens e a severidade total da amostra. A menor diferença entre os subgrupos e a amostra completa foi vista entre sexos, e a maior entre tipos de escolas (Figura 3).

Considerando que a Escala Brasileira de Medida de Insegurança Alimentar (EBIA), com 14 perguntas, entende como inseguro o domicílio que responde positivamente a pelo menos uma questão, no presente trabalho propôs-se uma análise da prevalência de insegurança alimentar com uma escala de cinco itens e pontos de corte adaptados, com comparabilidade à EBIA, incluindo, no grupo dos domicílios inseguros, os adolescentes que responderam a pelo menos um item positivamente. Dessa forma, foi considerado seguro somente o domicílio em que o adolescente não respondeu afirmativamente a nenhum item, e inseguro aquele em que o adolescente respondeu afirmativamente pelo menos um item. Os resultados mostraram que enquanto a prevalência de insegurança alimentar domiciliar medida pela escala curta com seis itens foi de $11,9 \%$, com a escala de cinco itens e pontos de corte adaptados, essa prevalência passou para $24,5 \%$ dos domicílios dos entrevistados.

\section{I S C U S S Ã O}

Este estudo forneceu evidências de que a escala de segurança alimentar reduzida com cinco itens é válida e confiável para mensurar a situação de insegurança alimentar domiciliar quando aplicada ao público adolescente.

Quanto à severidade dos itens, foi observado que o item cinco da escala original com 6 itens aparece com maior severidade do que o último item. Por se tratar de uma questão que mede a frequência do evento e não sua ocorrência, o item cinco naturalmente assume uma posição de maior gravidade do que a ocorrência de fome quando o evento de comer menos acontece com alta frequência. Dessa forma, quando a escala foi analisada sem esse item, a gravidade entre os outros itens foi crescente conforme espe- 


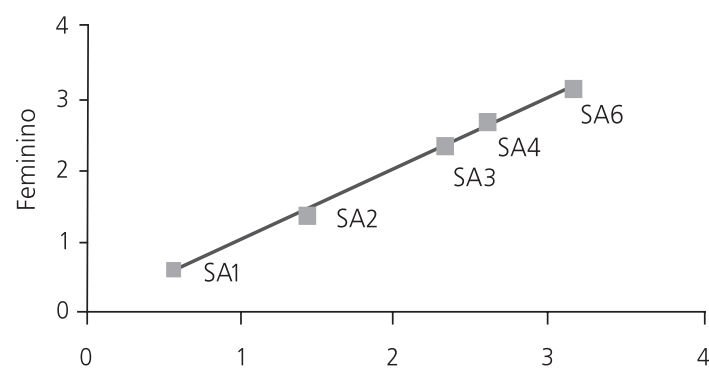

- Severidade total Severidade feminino ajustada

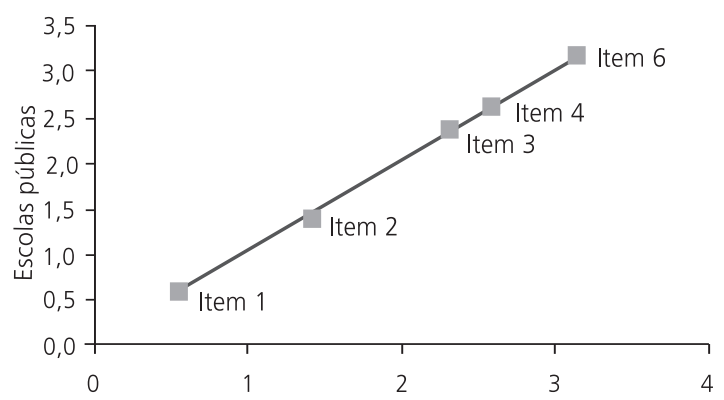

- Severidade total Severidade feminino ajustada

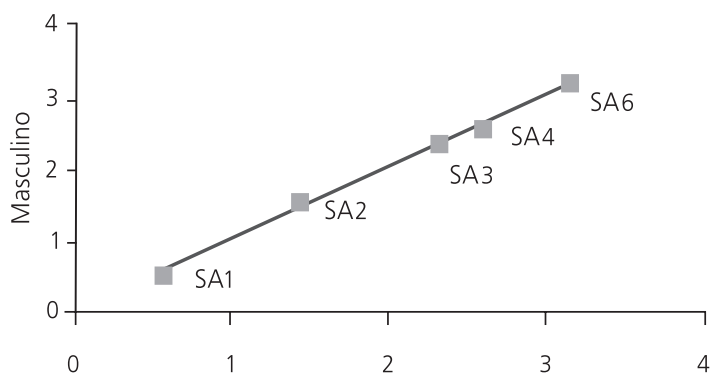

- Severidade total $\square$ Severidade feminino ajustada

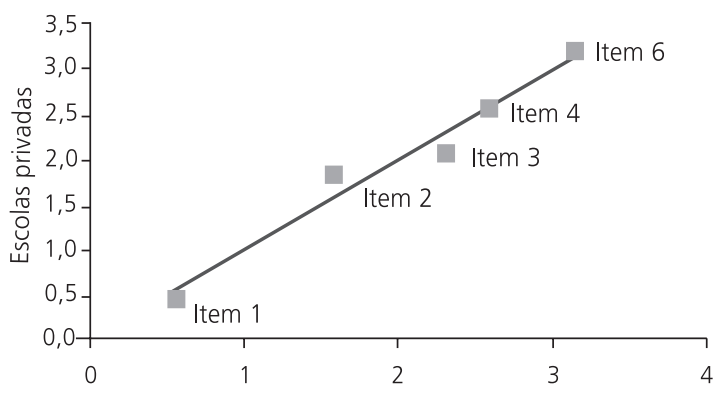

- Severidade total Severidade feminino ajustada

Figura 3. Comparação da severidade relativa dos itens da escala de segurança alimentar reduzida por sexo do adolescente: masculino ( $n=6$ 868) e feminino ( $n=7$ 822) versus total da amostra ( $n=14$ 690) e por tipo de escola que o adolescente frequenta: públicas $(n=10680)$ e privadas ( $n=4$ 006) versus total da amostra $(n=14690)$.

rado, desde a falta de uma alimentação variada até a ocorrência de fome ${ }^{7}$. Partindo dessa observação, e de que os ajustes da escala se tornavam ótimos com cinco itens, essa escala passou a ser considerada a ideal neste estudo e denominada aqui como Escala Curta de Insegurança Alimentar.

A Escala Curta de Insegurança Alimentar apresentou uma consistência interna aceitável com base no valor de alfa de Cronbach, um ajuste ótimo dos itens observado na análise de Rasch e comportamento similar de severidade dos itens em diferentes subgrupos, quando comparada à amostra completa.

Os valores de alfa de Cronbach tendem a aumentar com o número de itens de uma escala, e valores muito elevados sugerem que alguns itens podem ser redundantes, sendo ideal que alfa seja maior que $0,70^{22}$. Logo, a escala de segurança alimentar reduzida apresentará valores de alfa menores, porém aceitáveis, quando comparados à escala completa. Foi observado neste estudo um alfa de 0,77 , semelhante ao estudo com a mesma escala em adolescentes de Trinidad e Tobago, que encontrou o mesmo valor de alfa, indicando que o instrumento possui confiabilidade interna adequada para mensurar a insegurança alimentar ${ }^{10}$. Em estudo realizado com a EBIA, com 15 itens, foi observado um alfa de 0,93 e 0,90 para domicílios com e sem crianças, respectivamente ${ }^{23}$.

Os valores de Infit não ultrapassaram os valores mínimo e máximo $(0,7$ e 1,3), mostrando que a escala tem um bom ajuste entre a resposta estimada e a resposta esperada pelo modelo. Esse resultado foi semelhante ao observado por Gulliford et al. ${ }^{10} \mathrm{em}$ Trinindad e Tobado, com Infit entre 0,79 e 1,13 . Sem o item cinco, o ajuste passou a ser considerado ótimo, valores entre 0,8 e 
1,2. Os resultados mostraram que famílias com ocorrência de insegurança alimentar em maior gravidade respondem mais itens positivamente que famílias com menor gravidade ${ }^{19}$.

Os resultados também mostraram que a insegurança alimentar mensurada pela Escala Curta de Insegurança Alimentar foi mais prevalente quando o respondente era do sexo masculino, estudante de escola pública e filho de mãe com menor escolaridade. Essa associação já foi observada anteriormente em diversos estudos nacionais: a ocorrência de insegurança alimentar está associada à menor renda e escolaridade do chefe de família, que são fatores explicativos das condições acima referidas ${ }^{24-26}$.

Quanto à classificação do domicílio em seguro ou inseguro, a escala americana (HHFSM) da qual deriva a escala de seis itens é diferente da EBIA em dois aspectos. Primeiramente, a escala americana considera como seguros os domicílios que marcam até um ponto no escore ${ }^{7}$, enquanto no Brasil os domicílios são seguros somente quando não pontuam ${ }^{26}$. Dessa forma, a utilização da escala curta em sua classificação original poderia gerar uma subestimativa da real prevalência de insegurança alimentar entre os adolescentes quando comparada à aferição por meio da EBIA. Quando os dados da Escala Curta de Insegurança Alimentar foram analisados considerando como inseguras as famílias que responderam afirmativamente a pelo menos um item, a prevalência de insegurança alimentar na população estudada aumentou de 12,0 para 24,5\%. Esse resultado é mais próximo da prevalência de insegurança alimentar nos domicílios brasileiros com o grupo etário de 5 a 17 anos, observada pela Pesquisa Nacional por Amostra de Domicílios em 2009, que foi de $43,4 \%{ }^{26}$. Essa baixa prevalência de insegurança alimentar também pode ser justificada pelas características da população de estudo, formada por adolescentes residentes nas capitais dos estados brasileiros e que estudam em instituições com uma estrutura mínima para realização da pesquisa.
O segundo aspecto a ser considerado é que a Escala Curta de Insegurança Alimentar apenas permite comparação com a EBIA quando se trata de domicílios seguros ou inseguros, não sendo possível comparar os três níveis de insegurança alimentar. Enquanto a EBIA classifica o domicílio em seguro, e inseguro leve, moderado e grave, a Escala Curta de Insegurança Alimentar apenas permite classificar em seguro e inseguro. Sugere-se, portanto, novos pontos de corte para a Escala Curta de Insegurança Alimentar quando utilizada para a população brasileira, considerando que famílias que respondem afirmativamente a pelo menos um item da escala já são, pelo conceito brasileiro, consideradas com algum grau de insegurança alimentar.

Os pontos de corte sugeridos para Escala Curta de Insegurança Alimentar e baseados nas análises apresentadas são: entre 1 e 2 pontos, insegurança alimentar leve; entre 3 e 4, insegurança alimentar moderada; e 5 pontos, insegurança alimentar grave. Esses pontos de corte tornam possíveis comparações com a EBIA, embora dificultem comparações com estudos internacionais que utilizaram a escala Norte Americana com os seis itens.

É importante salientar que, apesar de as análises de validação terem sido realizadas sem a ponderação da amostra (o que não traz prejuízos para a validação), a amostra estudada é suficiente para garantir os resultados encontrados.

\section{CONSIDERAÇÕES FINAIS}

A escala de insegurança alimentar reduzida (Escala Curta de Insegurança Alimentar) é válida e confiável para ser utilizada em adolescentes brasileiros para mensurar insegurança alimentar domiciliar. O instrumento pode ser utilizado para rastreamento da insegurança alimentar e, por se tratar de um questionário reduzido, demanda pouco tempo para aplicação, além de baixo custo.

Por meio das análises realizadas, foi demonstrado que a escala curta original com 6 itens 
tem melhor funcionamento para os adolescentes brasileiros quando retirado o item cinco. Sugere-se, portanto, que a Escala Curta de Insegurança Alimentar utilizada no Brasil seja de apenas cinco itens. Com a utilização dos pontos de corte adaptados e sugeridos neste estudo, tornam-se possíveis comparações com estudos nacionais que utilizaram a EBIA como instrumento de mensuração da insegurança alimentar e evita subestimação da prevalência de insegurança alimentar.

Novos estudos poderão investigar a relação entre a insegurança alimentar vivenciada pelos adolescentes e as repercussões para a saúde e nutrição nessa fase da vida.

\section{A GRADECIMENTOS}

Ao Ministério da Educação e Secretarias de Educação Municipais e Estaduais de todos os 26 estados e do Distrito Federal o apoio prestado e pela viabilização da realização da coleta de dados.

\section{COLABORADORES}

SEAC COELHO contribuiu na concepção do projeto de pesquisa, análise e interpretação dos dados e redação do artigo. RPT VIANNA e AM SEGALL-CORRÊA participaram da análise e interpretação dos dados e revisão crítica do manuscrito. $R$ PEREZ-ESCAMILLA contribuiu na revisão crítica do manuscrito. MB GUBERT participou na concepção do projeto de pesquisa, redação do manuscrito e revisão crítica do conteúdo intelectual. Todos os autores participaram da aprovação final da versão a ser publicada.

\section{REFERÊ NCIAS}

1. Burity V, Franceschini T, Valente F. Segurança alimentar e nutricional e o direito humano à alimentação adequada. In: Direito Humano à Alimentação Adequada no contexto da Segurança Alimentar e Nutricional. Brasília: ABRANDH; 2010 [acesso 2013 dez 13]. Módulo 1. Disponível em: http://www. redsan-cplp.org/uploads/5/6/8/7/5687387/ manual_direito_humano_alimentao_adequada.pdf
2. Pérez-Escamilla R, Segall-Corrêa AM. Indicadores e medidas de insegurança alimentar. Rev Nutr. 2008; 21(Supl.):15-26.

3. Kepple AW, Segall-Corrêa AM. Conceituando e medindo segurança alimentar e nutricional. Ciência Saúde Colet. 2011; 16(1):187-99.

4. Jones $A D$, Ngure FM, Pelto G, Young SL. What are we assessing when we measure food security? A compendium and review of current metrics. Adv Nutr. 2013; 4(5):481-505.

5. Segall-Corrêa AM, Pérez-Escamilla R, Sampaio MFA, Marín-León L, Panigasse G, Kurdian Maranha L, et al. Relatório Técnico: versão preliminar. Acompanhamento e avaliação da segurança alimentar de famílias brasileiras: validação de metodologia e de instrumento de coleta de informação. Campinas: Unicamp; 2004.

6. Pérez-Escamilla R, Segall-Corrêa AM, Kurdian Maranha L, Sampaio MFA, Marín-León L, Panigasse $\mathrm{G}$, et al. An adapted version of the U.S. Department of Agriculture Food Insecurity Module is a valid tool for assessing household food insecurity in Campinas, Brazil. J Nutr. 2004; 134:1923-8.

7. Bickel GW, Nord M, Price C, Hamilton W, Cook J. Measuring food security in the United States. Guide to measuring household food security. Alexandria: United States Department of Agriculture; 2000 [cited 2013 Dec 13]. Available from: http:// www.fns.usda.gov/fsec/files/fsguide.pdf

8. Santos JV, Gigante DP, Domingues MR. Prevalência de insegurança alimentar em Pelotas, Rio Grande do Sul, Brasil, e estado nutricional de indivíduos que vivem nessa condição. Cad Saúde Pública. 2010; 26(1):41-9.

9. Gulliford MC, Mahabir D, Rocke B. Reliability and validity of a short form household food security scale in a Caribbean community. BMC Public Health. 2004; 4:22.

10. Gulliford MC, Mahabir D, Nunes C, Rocke B. Selfadministration of a food security scale by adolescents: Item functioning, socio-economic position and food intakes. Public Health Nutr. 2005; 8(7):853-60.

11. Vitolo MR. Hábitos alimentares na adolescência. In: Nutrição: da gestação ao envelhecimento. Rio de Janeiro: Editora Rubio; 2008. p.291-7.

12. Kac G, Velásquez-Melendez G, Schlüssel MM, Segall-Côrrea AM, Silva AA, Pérez-Escamilla R. Severe food insecurity is associated with obesity among Brazilian adolescent females. Public Health Nutr. 2012; 15(10):1854-60.

13. Pérez-Escamilla R, Vianna RPT. Food insecurity and the behavioral and intellectual development of 
children: A review of the evidence. J Applied Res Children; 2012; 3:1.

14. Rogol AD, Clark PA, Roemmich JN. Growth and pubertal development in children and adolescents: Effects of diet and physical activity. Am J Clin Nutr. 2000; 72(2 Suppl.):521S-8S.

15. Instituto Brasileiro de Geografia e Estatística. Censo demográfico: 2000. Rio de Janeiro: IBGE; 2000 [acesso 2013 dez 13]. Disponível em: http://www. ibge.gov.br/censo/default.php

16. Corrar LJ, Paulo E, Dias Filho JM. Análise multivariada. Fipecafi. São Paulo: Atlas; 2007.

17. Harrington D. Confirmatory factor analysis: Pocket guide to social work research methods. Oxford: Oxford University Press; 2009.

18. Laros JA. O uso da análise fatorial: algumas diretrizes para pesquisadores. In: Pasquali L. Análise fatorial para pesquisadores. Brasília: LabPAM; 2012. p.141-60.

19. Smith EV, Conrad KM, Chang K, Piazza J. An introduction to Rasch measurement for scale development and person assessment. J Nur Measurement. 2002; 10(3),189-206.

20. Bond TG, Fox CM. Applying the Rasch Model: Fundamental measurement in the human sciences. New Jersey: Lawrence Erlbaum Associates Inc. Publishers; 2001.

21. Derrickson JP, Fisher AG, Anderson JE. The core food security module scale measure is valid and reliable when used with Asians and Pacific Islanders. J Nutr. 2000; 130(11):2666-74.

22. Streiner DL, Norman GR. Health Measurement Scales: A practical guide to their development and use. $3^{\text {rd }}$ ed. Oxford: Oxford University Press; 2003.

23. Vianna RPT, Hromi-Fiedler AJ, Segall-Correa AM, Pérez-Escamilla R. Household food insecurity in small municipalities in Northeastern Brazil: A validation study. Food Security. 2012; 4:295-303.

24. Panigassi G, Segall-Corrêa AM, Marin-León L, PérezEscamilla R, Sampaio MFA, Maranha LK. Insegurança alimentar: um indicador de iniquidade. Cad Saúde Pública. 2008; 24(10):2376-84.

25. Salles-Costa R, Pereira RA, Vasconcellos MTL, Veiga GV, Marins VMR, Jardim BC, et al. Associação entre fatores socioeconômicos e insegurança alimentar: estudo de base populacional na Região Metropolitana do Rio de Janeiro, Brasil. Rev Nutr. 2008; 21(Supl.):99-109.

26. Instituto Brasileiro de Geografia e Estatística. Pesquisa Nacional por Amostra de Domicílios - PNAD: segurança alimentar 2004/2009. Rio de Janeiro: IBGE; 2010.
Recebido: setembro 29, 2014 Versão final: abril 28, 2015 Aprovado: maio 18, 2015 
